\title{
Bone development and remodeling in metabolic disorders
}

Jenny Serra-Vinardell ${ }^{1,2}$, Neus Roca-Ayats ${ }^{1}$, Laura De-Ugarte ${ }^{3,4}$, Lluïsa

Vilageliu ${ }^{1}$, Susanna Balcells ${ }^{1}$ and Daniel Grinberg ${ }^{1}$

${ }^{1}$ Department of Genetics, Microbiology and Statistics, Faculty of Biology,

Universitat de Barcelona, CIBERER, IBUB, IRSJD, Barcelona, Spain

${ }^{2}$ Section of Human Biochemical Genetics, Medical Genetics Branch, National

Human Genome Research Institute, National Institutes of Health, Bethesda, MD 20892, USA

${ }^{3}$ Department of Anatomy and Cell Biology, Indiana University School of

Medicine, Indianapolis, Indiana, United States of America

${ }^{4}$ Indiana Center for Musculoskeletal Health, Indianapolis, Indiana, United States of America

This is the author's manuscript of the article published in final edited form as:

Serra - Vinardell, J., Roca - Ayats, N., De - Ugarte, L., Vilageliu, L., Balcells, S., \& Grinberg, D. (2020). Bone development and remodeling in metabolic disorders. Journal of Inherited Metabolic Disease, 43(1), 133-144. https://doi.org/10.1002/jimd.12097 


\section{Abstract}

There are many metabolic disorders that present with bone phenotypes. In some cases, the pathological bone symptoms are the main features of the disease whereas in others they are a secondary characteristic. In general, the generation of the bone problems in these disorders is not well understood and the therapeutic options for them are scarce. Bone development occurs in the early stages of embryonic development where the bone formation, or osteogenesis, takes place. This osteogenesis can be produced through the direct transformation of the preexisting mesenchymal cells into bone tissue (intramembranous ossification) or by the replacement of the cartilage by bone (endochondral ossification). On the contrary, bone remodeling takes place during the bone's growth, after the bone development, and continues throughout the whole life. The remodeling involves the removal of mineralized bone by osteoclasts followed by the formation of bone matrix by the osteoblasts, that subsequently becomes mineralized. In some metabolic diseases bone pathological features are associated with bone development problems but in others they are associated with bone remodeling. Here we describe three examples of impaired bone development or remodeling in metabolic diseases, including work by others and results from our research. In particular, we will focus on hereditary multiple exostosis (or osteochondromatosis), Gaucher disease and the susceptibility to atypical femoral fracture in patients treated with bisphosphonates for several years. 


\section{Compliance with Ethics Guidelines}

Jenny Serra-Vinardell, Neus Roca-Ayats, Laura De-Ugarte, Lluïsa Vilageliu, Susanna Balcells and Daniel Grinberg declare that they have no conflict of interest. 


\section{Osteochondromatosis or multiple hereditary exostosis.}

Osteochondromatosis is characterized by the growth of multiple benign tumours mainly in long bones. The disease belongs to the group of congenital disorders of glycosylation. In particular, it is a defect of O-glycosylation. It is inherited as an autosomal dominant disease and it is caused by monoallelic mutations either in the EXT1 or in the EXT2 genes (reviewed in Wuyts and van Hul 2000). As a consequence of this, the alternative name for the disorder is EXT1/EXT2-CDG. The EXT1 (exostosin 1) and EXT2 (extosin 2) proteins form a co-polymerase involved in heparan sulfate biosynthesis.

The disease is related to bone development. Endochondral ossification is one of the two essential processes during fetal development of the mammalian skeletal system, by which bone tissue is created through the replacement of growing cartilage by bone (the other process is intramembranous ossification). In long bones there is a region, called the growth plate, in which chondrocytes proliferate and are replaced by osteoblasts. In this process, several signaling molecules play key roles in the regulation and direction of bone growth (Stickens and Evans 1998). Extracellular heparan sulfate makes a barrier that regulates the flux of these molecules. Individuals with an inherited mutation in one allele of EXT1 or EXT2 can suffer a second (somatic) mutation in the wildtype allele. This will lead to a local lack of heparan sulfate and an impaired regulation of bone growth, giving rise to osteochondromas (Bovee 2010). The role of heparan sulfate in the dis ease has been recently reviewed by Maurizio 
Pacifici (2018). The decrease in exostosin and heparan sulfate levels caused by the second hit (the somatic mutation in the wild-type allele of the EXT gene with a germline mutation in the other allele), causes a decrease in signaling molecules such as FGF, MEK, ERK, Noggin and Gremlin, and an increase of BMP and Hh signaling and of heparanase, which are involved in the abnormal growing of bone. For this reason, this disease can be considered as an example of abnormal bone development. The most severe complication of osteochondromatosis is malignant transformation of an osteochondroma into a peripheral secondary chondrosarcoma. The estimated lifelong risk varies among different studies from $1 \%$ to $25 \%$ (Beltrami et al. 2016). Czajka and DiCaprio (2015) studied a large international, heterogeneous cohort of around 800 patients with multiple hereditary exostoses and reported a proportion of $2.7 \%$ of malignant degeneration to chondrosarcoma. The mechanisms for this malignant change are not clear. Different authors reported genetic mutations in genes different from EXT1 and EXT2 during chondrosarcoma progression and it is assumed that these are necessary to progress into malignancy (Musso et al. 2015, and references therein). Musso et al. (2015) described a surprising case in which they observed a loss of the EXT2 mutant allele in the peripheral secondary chondrosarcoma, instead of the expected loss of the EXT2 wild-type allele, suggesting a different cell of origin for osteochondromas and chondrosarcomas. However, this is a topic that is still open. 
Our group has studied the mutations present in Spanish (Sarrion et al. 2013) and Latino American (Delgado et al. 2012, 2014, Cammarata-Scalisi et al. 2015, 2018) patients. In general, we sequenced PCR-amplified exons and flanking regions of the EXT1 and EXT2 genes, followed by MLPA analysis if the sequencing of the exons gave negative results. In some cases, we observed mosaicism in the first affected individual of the family (Sarrion et al. 2013). Occasionally, one of the parents of the proband, with a very mild phenotype, learned about his/her disease after our analysis of the family. In one particular case, a patient came to us after having obtained negative results both of sequencing and MLPA analyses by a private molecular diagnosis company. We thought that she could either bear a hidden mutation in EXT1 or EXT2, or bear a mutation in a hypothetical EXT3 gene, suggested to exist but never found. We resequenced the EXT1 and EXT2 genes and with some of the SNPs found in the resequencing we performed a segregation analysis in the patient's family to see if we were able to discard the involvement of these two genes in their disease. The result was the opposite: the segregation analysis showed an apparent lack of heredity of EXT2, only consistent with a deletion (Fig. 1a, 1b), which we demonstrated afterwards by MLPA (Fig. 1c). It was a serious mistake of the private company.

As mentioned above, osteochondromatosis is inherited as an autosomal dominant disease. Thus, only a mutation in one allele of either EXT1 or EXT2 is present in the germline. Until a few years ago, no case with germline mutations 
in the two alleles of these genes was known. However, two cases with homozygous germline missense mutations in the EXT2 gene have been recently published (Farhan et al. 2015, El-Bazzal et al. 2019). Surprisingly, the phenotype is characterized by seizures and developmental disorders without exostoses. The only bone phenotype was osteopenia, present in one of the patients. The proposed name for this novel syndrome is autosomal recessive EXT2-related syndrome (AREXT2). Notably, these cases show that the consequences of these homozygous missense mutations are critical for brain development, while not affecting bone. The role of heparan sulfate in the brain is not well understood but very recently, it was shown that it organizes neuronal synapses through neurexin partnerships (Zhang et al. 2018). Further research will be necessary to better understand the dual roles of EXT2 in bone and brain.

We have also studied the family of EXT genes from a different point of view. In Sanfilippo disease (mucopolysaccharidosis III) there is an accumulation of heparan sulfate in the lysosomes due to an impaired function of one of several lysosomal enzymes. We have assayed the inhibition of the EXTL2 and EXTL3 genes, as a substrate reduction therapy for Sanfilippo $\mathrm{C}$ disease in patients' fibroblasts (Canals et al. 2015a) and we are currently performing a similar approach on Sanfilippo C neurons, derived from iPSC generated by our group (Canals et al. 2015b).

\section{Gaucher disease}


Lysosomal storage diseases (LSDs) are a group of more than 50 disorders, which mainly result from the deficient activity of specific lysosomal enzymes. This deficiency produces a progressive accumulation of specific substrates affecting different biochemical or cellular pathways, which subsequently will cause the tissue pathology (Futerman and van Meer 2004). Gaucher disease (GD), the most common LSD, is caused by mutations in the GBA1 gene (MIM\# 606463) that produce a defective activity of glucocerebrosidase (EC 3.2.1.45; GBA1), the lysosomal enzyme responsible for the hydrolysis of glucosylceramide (GlcCer) into glucose and ceramide. As a result of this autosomal recessive genetic defect, GlcCer and glucosylsphingosine (GlcSph) accumulate in the lysosomes of macrophages (revised by Sidransky 2004) generating the typical "Gaucher cells", the hallmark of the disease. Based on the absence or presence and severity of neuronopathic involvement, GD has been classified into three clinical phenotypes, non-neuronopathic (GD1), acute neuronopathic (GD2) and chronic or subacute neuronopathic (GD3) (Beutler and Grabowski 1995). An extreme phenotypic variability has been reported for Gaucher disease, within each of the clinical types, and even among patients with the same mutations. This variability is likely due to a multitude of factors such as genetic background, environment, and epigenetic status (Sidransky 2004). Davidson et al. (2018) reviewed genetic modifiers that influence the phenotypic outcome of Gaucher disease. 
GD1 is the most frequent form of the disease and it is characterized by heterogeneous manifestations including visceral (hepatosplenomegaly), hematological and skeletal symptoms. Bone involvement affects up to $90 \%$ of GD1 patients and it is for them the most debilitating feature because it has a major impact on their life quality (Giraldo 2005). Skeletal manifestations include erlenmeyer flask deformity, fractures due to osteopenia or osteoporosis, osteosclerosis, osteonecrosis, bone pain, bone crisis, growth retardation during childhood and, rarely, acute osteomyelitis (Mikosch and Hughes 2010). The majority of these manifestations could be explained by the disruption of the balance between osteoblastic bone formation and osteoclastic bone resorption. The markers of bone metabolism are useful to measure changes in the activities of osteoblasts and osteoclasts. However, controversial results on the alteration of bone formation and of bone resorption markers in GD and their response to enzyme therapy have been reported (revised by van Dussen et al. 2011). Furthermore, we do not have a conclusive statement from previous studies where mouse or cell models were used to understand bone pathology in GD. Basically, the lack of consistence between studies is an evidence that the bone pathology in GD is complex and, based on the wide phenotypic spectrum in patients, may be a pleiotropic disease.

Campeau et al. (2009) revealed that Mesenchymal Stromal Cells (MSCs) from a GD1 patient (N370S/L444P genotype) displayed an altered secretome that may contribute to the skeletal and immune problems in GD. Afterwards, 
Mistry et al. (2010) provided an important model, using a conditional GBA1 knockout in hematopoietic and mesenchymal cell lineages. They reported that the model recapitulated the main features of GD1, such as visceral and hematologic diseases together with a profound osteopenia. In addition, they provided evidences that whereas the mouse model had a normal osteoclast formation (osteoclastogenesis), the bone formation (osteoblastogenesis) appeared to be defective. They suggested that the osteoblastogenesis was inhibited by the accumulation of the lipids GlcCer and GlcSph, and their consequent interaction with the protein kinase $\mathrm{C}(\mathrm{PKC})$. Later on, the same authors proposed that the extralysosomal glucocerebrosidase GBA2 transformed the increased levels in serum of GlcCer and, mainly, GlcSph in sphingosine, which inhibited osteoblasts survival (Mistry et al. 2014). Despite of these previous evidences, other studies confirmed a correlation between bone features and osteoclast number in GD (Bondar et al. 2017; Mucci et al. 2012, 2013; Reed et al. 2013, 2018).

Several groups decided to use human induced Pluripotent Stem Cells (iPSC)-derived osteoblasts to address the unknown of the bone pathology in GD. The main study so far is the one recently published by Ricardo Feldman's group (Panicker et al. 2018). They showed that GD iPSC-derived osteoblasts had developmental and lysosomal defects that impaired bone matrix deposition. Moreover, they showed that the canonical Wnt pathway was affected. In concordance, many studies showed the importance of this pathway in bone 
metabolism, including ours (Estrada et al. 2012, Zheng et al. 2015, Martínez-Gil et al. 2018).

The clinical bone manifestations in GD are included in the bone remodeling problems category, since bone problems appear as a post-natal trait. Whether the osteoblasts are downregulated or the osteoclasts are upregulated is one of the main questions that needs to be answered in order to look for an effective treatment.

Our aim was to create osteoblasts derived from wild-type hiPSC generated in our group (Canals et al. 2015b) and compare them with those generated from GD patients [N370S/N370S, a gift from Ricardo Feldman, G202R/L444P, a gift from Gustavo Tiscornia (Tiscornia et al. 2011), and N370S/84GG (Park et al., 2008)]. For the MSC-like cells induction we used a multistep culture method, summarized in Fig. 2a. For each MSC induction (WT, N370S/N370S, G202R/L444P, N370S/84GG) the expression of specific MSC surface markers (CD73, CD90, CD105) and the absence of expression of hematopoietic markers (CD34 and CD45) were verified (data not shown). This qualitative step is required to continue with the subsequent experiments. From now on, the MSC-like cells will be mentioned as MSC.

The MSCs with G202R/L444P and N370S/84GG genotypes presented lack of proliferation capacity and therefore could not be used in subsequent experiments. These cells were larger and flatter compared to the WT and N370S/N370S MSC (spindle-shaped cells in both cases). The lack of self- 
renewal of these large flattened cells has been previously described in the literature (Colter et al. 2000; Digirolamo et al. 1999). Because the relationship between cell size, morphology and senescence is well known (Bayreuther et al, 1988; von Zglinicki et al, 2003), we evaluated the cell cycle profile in the MSC stage (in WT, N370S/N370S and N370S7/84GG genotypes) by flow cytometry as an attempt to investigate the possible alteration of the cell cycle in GD cells. As shown in Fig. 2b, there was a remarkable proportion (98\%) of GD N370S/84GG cells arrested in G0/G1 phase compared to WT cells (88\%). Concomitant with this, there was a reduction in the number of replicative cells (phase S, N370S/84GG MSC $=0.6 \%$ compared to WT MSC $=2.9 \%$ ) and in the G2/M subpopulation (N370S/84GG MSC $=1.5 \%$ compared to WT $=8.7 \%$ ). Regarding the other GD genotype (N370S/N370S), the number of cells in S phase had also notably decreased in comparison to WT cells (N370S/N370S MSC $=0.9 \%$, WT MSC $=2.9 \%$ ). The classic features characterizing the senescence phenotype of MSCs include growth arrest in the G1 phase of the cell cycle, enlarged or flattened morphology and increased expression of senesce-associated $\beta$-galactosidase (not evaluated because the $\beta$ galactosidase activity in LSD is deregulated and could drive to misinterpretation). Thus, only GD cells with the N370S/N370S genotype were used in further experiments.

To check the GBA1 activity before and after the differentiation process we analyzed the enzyme activity in the MSC stage (considered as day 0) and 
after 21 days of the osteogenic differentiation. We showed that both, on day 0 and day 21 , the activity of GBA1 in GD cells was less than $15 \%$ (GD day $0=$ $13 \%$, GD day $21=14 \%$ ) compared to that of the WT cells. Moreover, the difference between GBA1 activity in WT and GD cells did not change during the differentiation process (Fig 2c).

We aimed to evaluate in vitro the potential impact of GBA1 deficiency on two representative genes of the osteogenic development and mineralization process, runt-related transcription factor 2 (RUNX2) and tissue-nonspecific alkaline phosphatase $(A L P L)$, by real time polymerase chain reaction (RTPCR). The expression of $A L P L$ during the differentiation was significantly higher in GD compared to WT cells (Fig. 3a) Although this is in disagreement with other authors who found the expression of this gene lower in GD compared to WT (Mistry et al. 2010, Panicker et al. 2018), other studies have reported no significant differences in the mRNA levels of this gene between MSCs of GD and WT (Compeau et al, 2009). In the same direction, Lecourt et al. (2012) showed that the activity of this enzyme did not change in CBE-treated MSCs, indicating that the inhibition of the GBA1 enzyme does not impact in the expression of $A L P L$. Regarding RUNX2 expression along differentiation, higher levels were observed at day 7 in both genotypes (GD and WT), corresponding to the MSC to osteoblasts transition. At all time-points, the expression of RUNX2 was higher in WT cells (Fig. 3b). 
To evaluate the osteoblast functionality and therefore the mineralization of the extracellular matrix, we confirmed the existence of hydroxyapatite crystals (the bone mineral content) by the staining of calcium (Fig. 4a) and phosphate (Fig. 4b). Both, calcium and phosphate were observed from day 14 in WT and GD cells.

The results of these experiments indicate that GD MSC seem to have a normal behavior, and they may differentiate properly to functional osteoblasts, in agreement with the model generated by Lecourt et al. (2012). We can assume that the GD cells have a normal bone development, as expected, since bone problems in GD are due to defects in bone remodeling and not in bone development. The differences between WT and GD in the expression of RUNX2 and $A L P L$ seem not to affect the function of GD osteoblasts.

Since osteoblast function was unaffected, GD bone pathology could be due to an increase in osteoclasts formation (osteoclastogenesis) or function. Osteoclats are multinucleated, tartrate resistant acid phosphatase (TRAP) positive and bone resorbing giant cells derived from the differentiation and fusion of mononuclear hematopoietic progenitors' cells in the monocyte/macrophage lineage. The osteoblast-lineage cells (MSC, preosteoblasts, osteoblasts and osteocytes) play an essential role in the regulation of osteoclastogenesis.

Osteoblast lineage cells and osteoclasts have a permanent crosstalk through molecules such as the receptor activator of the nuclear factor kappa B 
(RANK) and its ligand (RANKL). Osteoblasts lineage cells express in their surface and release RANKL, that binds to the receptor RANK in the preosteoclasts' surface and promote osteoclasts' differentiation, activation and survival. We performed co-cultures of MSC or osteoblasts derived either from wild-type or GD iPSC with monocytes from healthy donors (Fig.5a). The objective was to assess whether the osteoclastogenesis, promoted by the WT or GD iPSC-derived MSC/osteoblasts, was similar. With 4',6-diamidino-2phenylindole (DAPI) and TRITC-phalloidin, we were able to compare the nuclei number and the size of the osteoclasts generated by the two osteoblastic lineages (Fig. 5b). The cells need to have at least three nuclei to be considered mature osteoclasts. The TRAP staining of these cells revealed the osteoclast identity (Fig. 5c). The osteoclasts generated by GD iPSC-derived MSC/osteoblasts were larger than the ones generated by WT MSC/osteoblasts (Fig. 5d). However, there were no differences in the number of nuclei per osteoclasts (Fig. 5e), indicating that the larger osteoclasts are probably due to an increased spreading, rather than to an increment of cell fusion. We also analyze TRAP released into the culture media because it has been reported that it is in good correlation with the number of mature osteoclasts generated (Alatalo et al, 2000). However, we did not find consistent differences between the values in both conditions, assuming that the number of osteoclasts generated either by WT or GD iPSC-derived MSC/osteoblasts was similar (data not shown). 
In summary, the results presented here show that we were able to generate models of MSC and osteoblasts from GD- (and WT)-iPSC. However, it seems that these models do not reproduce faithfully the bone pathology in GD. Maybe the complexity of the bone pathology in this disease cannot be explained with cell-autonomous models. Similar to what had happened with previous cellular models, our model did not allow the evaluation of the direct cross-talking with other cells and other molecules such as the cytokines, which are known to be very important in the bone remodeling.

\section{Atypical femoral fracture}

The last example is that of a particular type of bone fracture, known as atypical femoral fracture (AFF). AFFs were defined as atraumatic or low-trauma fractures located in the subtrochanteric region or femoral shaft. The diagnosis of AFF specifically excludes high trauma fractures, fractures of the femoral neck, intertrochanteric fractures with spiral subtrochanteric extension, pathological fractures associated with primary or metastatic bone tumors, and periprosthetic fractures. The fractures are usually not comminuted. Other characteristic radiographic features of AFFs (Fig. 6a) include a transverse fracture line at the point of origination in the lateral cortex. As the fracture propagates across the diaphysis to the medial cortex, the orientation may become more oblique and when it becomes complete, a prominent medial "spike" may be present. There may be a focal or diffuse periosteal reaction of the lateral cortex surrounding the 
region where the fracture initiated. This reaction may appear as cortical "beaking" or "flaring" adjacent to a discrete transverse lucent fracture line or as focal thickening of the lateral cortex. Focal and diffuse endosteal reactions near the fracture site have been reported more recently. This focal cortical thickening represents cortical hypertrophy and may be unilateral or bilateral. There may also be generalized cortical thickening (Shane et al. 2014). Some epidemiological studies have suggested a relationship between AFFs and longterm bisphosphonates (BPs) therapy, the main treatment for osteoporosis (Gedmintas et al. 2013; Liu et al. 2015). Denosumab treatment has also been related to AFFs (reviewed in Anastasilakis et al 2018), although this relationship is not so clear. In any case, the pathogenesis of AFFs has not yet been elucidated.

We studied three sisters who had atypical femoral fractures after receiving various oral bisphosphonates for 6 years (Roca-Ayats et al. 2017). Two of the sisters had a single fracture and one had bilateral fractures. Given the low incidence of atypical femoral fractures in the general population (3.0-9.8 cases per 100,000 person-year, Khow et al. 2017), we hypothesized that these sisters might have an underlying genetic background that contributed to these fractures.

We performed whole-exome sequencing in the three sisters and in three unrelated patients with atypical femoral fractures who each had received bisphosphonates for more than 5 years. We prioritized rare nonsynonymous 
mutations in the variant filtering, and only mutations that were shared among the three sisters were considered. No mutation was found to be homozygous or in compound heterozygosity.

Assuming that a dominant model was involved, we detected 37 rare mutations (in 34 genes), among them a novel p.Asp188Tyr substitution in the enzyme geranylgeranyl pyrophosphate synthase (GGPPS). This variant had the best conservation score and was not described in any of the available population databases. Interestingly, GGPPS is a homohexameric enzyme (Kavanagh et al. 2006a) that participates in the mevalonate (or isoprenoid) pathway (Fig. 6b), catalyzing a reaction just downstream of the main site of inhibition by bisphosphonates (Kavanagh et al. 2006b). The mevalonate pathway leads to the production of cholesterol and isoprenoid lipids, such as farnesyl diphosphate (FPP) and geranylgeranyl diphosphate (GGPP), required for the post-translational prenylation of some proteins, including small GTPases. Prenylation of small GTPases is necessary for their activation and essential for osteoclast function and survival (Itzstein et al. 2011).

We performed in vitro functional analyses of the GGPPS p.Asp188Tyr mutation, which showed a severe reduction in enzyme activity together with mild oligomerization defects (Roca-Ayats et al. 2018b). Interestingly, another work on GGPPS p.Asp188Tyr mutation has been recently published (Lisnyansky et al. 2018) and the authors also observed a decreased catalytic activity of the mutated GGPPS, consistent with our results. In addition, they 
showed that it is unable to support cross-species complementation. On the contrary, they only observed hexameric conformation of the enzyme in crystallographic experiments, although they saw a slight break of the tertiary symmetry, as well as a lower thermal stability of the mutated enzyme. In addition, the new tyrosine residue sterically interferes with substrate binding. Moreover, and considering that GGPPS can also be inhibited by BPs, although to a lesser extent (Guo et al. 2007), they studied the affinity of the GGPPSD188Y for zolendronate, a commonly used BP, demonstrating that it exhibited a reduction in the binding affinity although it could still be inhibited by zolendronate.

We also performed cellular functional assays, such as RNAi knockdown of the GGPS1 gene in osteoblasts and in osteoclasts. In osteoblasts, RNAi produced a strong mineralization reduction and a reduced expression of the typical osteoblastic markers osteocalcin, osterix, and RANKL, whereas in osteoclasts, it led to an increase of osteoclast number with a lower resorption activity (Roca-Ayats et al. 2018b).

We and others have shown that GGPPS p.Asp188Tyr mutation has an impact on protein function and relevant effects on bone cells, making it a strong candidate for AFF susceptibility. We propose that excessive inhibition of osteoclastic activity by the mutation plus BPs may lead to reduced bone remodeling and toughness, which may increase AFF susceptibility. 
Few studies aiming to elucidate the genetics underlying AFF have been carried out, identifying some genes involved in AFF (Table 1). One of the most interesting genes is CYP1A1, an enzyme involved in the metabolism of drugs and xenobiotics and responsible for the hydroxylation of steroid hormones, such as estrogens (Zhou et al. 2009; Fig. 6b). Mutations in CYP1A1 were found in the 3 sisters and one unrelated patient in our study (Roca-Ayats et al. 2017, 2018b) and also reported elsewhere in two patients with AFF and glucocorticoid-induced osteoporosis (Peris et al. 2018). Another study identified a missense variant in the PPEF2 gene significantly associated with AFF by exon array analysis of a small cohort (Pérez-Núñez et al. 2015). To date, this gene has no known function in bone metabolism. Some studies have identified genes previously known to be involved in monogenetic bone diseases. Recently, Funck-Brentano and colleagues (2017) identified a heterozygous mutation in COL1A2, coding for type 1 collagen, a major component of the bone extracellular matrix. Mutations in COL1A2 cause osteogenesis imperfecta (OI). However, no specific physical features of Ol were identified in this patient, apart from short stature. In addition, Lau et al. (2017) identified a homozygous mutation in CTSK gene in a consanguineous family, encoding for cathepsin $\mathrm{K}$ and related to pycnodysostosis, although the patient identified had no clinical features of this disease. Finally, some cohort studies identified AFFs in patients of hypophosphatasia initially misdiagnosed with postmenopausal osteoporosis and treated with BPs (Peris et al. 2018, Sum et al. 2013). Heterozygous 
mutations in tissue non-specific alkaline phosphatase (TNSALP; ALPL gene) were identified. In these cases, it might be that mild, unrecognized forms of some monogenetic bone diseases underlie the etiology of AFFs. Taken together, all the genetic studies carried out in AFF patients advocate a heterogeneous genetic component of predisposition to AFF, in which each individual patient would be a carrier of different specific genetic variant/s (Fig. 6c). In addition, in our study we identified some other interesting rare variants in the 3 sisters and unrelated patients (e.g. MMP9, MVD, RUNX2, NGEF, SYDE2, FN1; Roca-Ayats et al. 2018a), suggesting a polygenic or complex background involving, to different extent, several additional variants in the susceptibility to AFF. All in all, we speculate that an accumulation of a few susceptibility variants from different pathways and their interactions constitute the genetic predisposition that, together with BPs and/or other comorbid conditions, give rise to AFF, in what we might call "the perfect storm". Further identification and/or replication of genetic variants, as well as functional studies of the identified variants, are needed to detect at-risk individuals for clinical decisionmaking.

\section{Acknowledgements}

The authors are grateful to M. Cozar for technical assistance and to patient DR and her family for collaboration and permanent support. JSV was a recipient of an FI fellowship from the Catalan government and NRA was a recipient of an 
FPU fellowship from the Spanish government. This work was supported by the following grants: SAF2016-75948-R (Spanish MINECO), 2014 SGR932 (Generalitat de Catalunya) and CIBERER (U720).

\section{References}

Alatalo SL, Halleen JM, Hentunen TA et al (2000) Rapid screening method for osteoclast differentiation in vitro that measures tartrate-resistant acid phosphatase $5 \mathrm{~b}$ activity secreted into the culture medium. Clin Chem 46(11):1751-1754

Anastasilakis AD, Polyzos SA, Makras P (2018) Therapy of endocrine disease: Denosumab vs bisphosphonates for the treatment of postmenopausal osteoporosis. Eur J Endocrinol 179(1):R31-R45

Bayreuther K, Rodemann HP, Hommel R, et al (1988) Human skin fibroblasts in vitro differentiate along a terminal cell lineage. Proc Natl Acad Sci U S A 85(14):5112-5116

Beltrami G, Ristori G, Scoccianti G, et al (2016) Hereditary Multiple Exostoses: a review of clinical appearance and metabolic pattern. Clin Cases Miner Bone Metab 13(2):110-118

Beutler E, Grabowski GA (1995) Gaucher disease. In Scriver CR, Beaudet AL, Sly WS, Valle D (eds): The Metabolic and Molecular Bases of Inherited Disease. 7th Ed. New York: McGraw-Hill, pp 2641-2669 
Bondar C, Mucci J, Crivaro A et al (2017) In vitro osteoclastogenesis from Gaucher patients' cells correlates with bone mineral density but not with Chitotriosidase. Bone 103:262-269

Bovee JVMG (2010) EXTra hit for mouse osteochondroma. Proc Natl Acad Sci U S A 107(5):1813-1814

Cammarata-Scalisi F, Cozar M, Grinberg D et al (2015) Double mutant alleles in the EXT1 gene not previously reported in a teenager with hereditary multiple exostoses. Arch Argent Pediatr 113(2):e109-112

Cammarata-Scalisi F, Stock F, Avendaño A et al (2018) Clinical and molecular study in a family with multiple osteochondromatosis. Acta Ortop Mex 32(2):108-111

Campeau PM, Rafei M, Boivin MN et al (2009) Characterization of Gaucher disease bone marrow mesenchymal stromal cells reveals an altered inflammatory secretome. Blood 114(15):3181-3190

Canals I, Benetó N, Cozar M et al (2015a) EXTL2 and EXTL3 inhibition with siRNAs as a promising substrate reduction therapy for Sanfilippo C syndrome. Sci Rep 5:13654

Canals I, Soriano J, Orlandi JG et al (2015b) Activity and High-Order Effective Connectivity Alterations in Sanfilippo C Patient-Specific Neuronal Networks. Stem Cell Reports 5(4):546-57 
Colter DC, Class R, DiGirolamo CM et al (2000) Rapid expansion of recycling stem cells in cultures of plastic-adherent cells from human bone marrow. Proc Natl Acad Sci U S A 97(7):3213-3218

Czajka CM, DiCaprio MR (2015) What is the proportion of patients with multiple hereditary exostoses who undergo malignant degeneration? Clin Orthop Relat Res 473(7):2355-2361

Davidson BA, Hassan S, Garcia EJ et al (2018) Exploring genetic modifiers of Gaucher disease: The next horizon. Hum Mutat 39(12):1739-1751

Delgado MA, Martinez-Domenech G, Sarrión P et al (2014) A broad spectrum of genomic changes in latinamerican patients with EXT1/EXT2-CDG. Sci Rep 4:6407

Delgado MA, Sarrión P, Azar N et al (2012) A novel nonsense mutation of the EXT1 gene in an Argentinian patient with multiple hereditary exostoses: a case report. J Bone Joint Surg Am 94(11):e76

Digirolamo CM, Stokes D, Colter D et al (1999) Propagation and senescence of human marrow stromal cells in culture: a simple colony-forming assay identifies samples with the greatest potential to propagate and differentiate. Br J Haematol 107(2):275-281

El-Bazzal L, Atkinson A, Gillart AC et al (2019) A novel EXT2 mutation in a consanguineous family with severe developmental delay, microcephaly, seizures, feeding difficulties, and osteopenia extends the phenotypic 
spectrum of autosomal recessive EXT2-related syndrome (AREXT2). Eur J Med Genet 63(4):259-264

Estrada K, Styrkarsdottir U, Evangelou E et al (2012) Genome-wide metaanalysis identifies 56 bone mineral density loci and reveals 14 loci associated with risk of fracture. Nat Genet 44(5):491-501

Farhan SM, Wang J, Robinson JF et al (2015) Old gene, new phenotype: mutations in heparan sulfate synthesis enzyme, EXT2 leads to seizure and developmental disorder, no exostoses. J Med Genet 52(10):666-675

Funck-Brentano T, Ostertag A, Debiais F, et al (2017) Identification of a p.Arg708GIn variant in COL1A2 in atypical femoral fractures. Jt Bone Spine 84:715-718

Futerman AH, van Meer G (2004) The cell biology of lysosomal storage disorders. Nat Rev Mol Cell Biol 5(7):554-565

Gedmintas L, Solomon DH, Kim SC (2013) Bisphosphonates and risk of subtrochanteric, femoral shaft, and atypical femur fracture: A systematic review and meta-analysis. J Bone Miner Res 28:1729-1737

Giraldo P, Solano V, Pérez-Calvo Jl et al (2005) Quality of Life Related to Type 1 Gaucher Disease: Spanish Experience. Qual Life Res 14(2):453-462

Guo R-T, Cao R, Liang P-H, et al (2007) Bisphosphonates target multiple sites in both cis- and trans-prenyltransferases. Proc Natl Acad Sci U S A 104:10022-10027 
Itzstein C, Coxon FP, Rogers MJ (2011) The regulation of osteoclast function and bone resorption by small GTPases. Small GTPases 2:117-130

Kavanagh KL, Dunford JE, Bunkoczi G, et al (2006a) The crystal structure of human geranylgeranyl pyrophosphate synthase reveals a novel hexameric arrangement and inhibitory product binding. J Biol Chem 281:2200422012

Kavanagh KL, Guo K, Dunford JE, et al (2006b) The molecular mechanism of nitrogen-containing bisphosphonates as antiosteoporosis drugs. Proc Natl Acad Sci 103:7829-7834

Khow KSF, Shibu P, Yu SCY, et al (2017) Epidemiology and postoperative outcomes of atypical femoral fractures in older adults: A systematic review. J Nutr Heal Aging 21:83-91

Lau S-L, Mclnerney-Leo AM, Lanoda-Bassonga E et al (2017) Homozygous variant in Cathepsin $\mathrm{K}$ in a family with multiple cases of bilateral atypical femoral fracture but without clinical features of pyknodysostosis. JBMR Plus 1(S1):S1-S11

Lecourt S, Vanneaux V, Cras A et al (2012) Bone Marrow Microenvironment in an in Vitro Model of Gaucher Disease: Consequences of Glucocerebrosidase Deficiency. Stem Cells Dev 21(2):239-248

Lisnyansky M, Kapelushnik N, Ben-Bassat A, et al (2018) Reduced Activity of Geranylgeranyl Diphosphate Synthase Mutant Is Involved in 
Bisphosphonate-Induced Atypical Fractures. Mol Pharmacol 94:13911400

Liu L, Li C, Yang P, et al (2015) Association between alendronate and atypical femur fractures: a meta-analysis. Endocr Connect 4:58-64

Martínez-Gil N, Roca-Ayats N, Monistrol-Mula A et al (2018) Common and rare variants of WNT16, DKK1 and SOST and their relationship with bone mineral density. Sci Rep 8(1):10951

Mikosch P, Hughes D (2010) An overview on bone manifestations in Gaucher disease. Wien Med Wochenschr 160(23-24):609-624

Mistry PK, Liu J, Sun L et al (2014) Glucocerebrosidase 2 gene deletion rescues type 1 Gaucher disease. Proc Natl Acad Sci U S A 111(13): 49344939

Mistry PK, Liu J, Yang M et al (2010) Glucocerebrosidase gene-deficient mouse recapitulates Gaucher disease displaying cellular and molecular dysregulation beyond the macrophage. Proc Natl Acad Sci U S A 107(45):19473-19478

Mucci JM, Scian R, De Francesco PN et al (2012) Induction of osteoclastogenesis in an in vitro model of Gaucher disease is mediated by T cells via TNF- $\alpha$. Gene 509(1):51-59

Mucci JM, Suqueli García F, de Francesco PN et al (2013) Uncoupling of Osteoblast-Osteoclast Regulation in a Chemical Murine Model of Gaucher Disease. Gene 532(2):186-191 
Musso N, Caronia FP, Castorina S et al (2015), Somatic loss of an EXT2 gene mutation during malignant progression in a patient with hereditary multiple osteochondromas. Cancer Genet 208(3):62-67

Pacifici M (2018) The pathogenic roles of heparan sulfate deficiency in hereditary multiple exostoses. Matrix Biol 71-72:28-39

Panicker LM, Srikanth MP, Castro-Gomes T et al (2018) Gaucher disease iPSC-derived osteoblasts have developmental and lysosomal defects that impair bone matrix deposition. Hum Mol Genet 27(5):811-822

Park IH, Arora N, Huo H et al (2008) Disease-specific induced pluripotent stem cells. Cell 134(5):877-886

Pérez-Núñez I, Pérez-Castrillón JL, Zarrabeitia MT, et al (2015) Exon array analysis reveals genetic heterogeneity in atypical femoral fractures. A pilot study. Mol Cell Biochem 409:45-50

Peris P, González-Roca E, Rodriguez-García SC, et al (2019) Incidence of Mutations in the ALPL, GGPS1, and CYP1A1. JBMR Plus 3(1):29-36

Reed M1, Baker RJ, Mehta AB et al (2013) Enhanced differentiation of osteoclasts from mononuclear precursors in patients with Gaucher disease. Blood Cells Mol Dis 51(3):185-194

Reed MC, Bauernfreund $Y$, Cunningham N et al (2018) Generation of osteoclasts from type 1 Gaucher patients and correlation with clinical and genetic features of disease. Gene 678:196-206 
Roca-Ayats N, Balcells S, Garcia-Giralt N et al (2017) GGPS1 Mutation and Atypical Femoral Fractures with Bisphosphonates. N Engl J Med 376(18):1794-1795

Roca-Ayats N, Falcó-Mascaró M, Garcia-Giralt N et al (2018a) Genetic study of atypical femoral fractures using exome sequencing in three affected sisters and three unrelated patients. Rev Osteoporos Metab Miner 10(4):108-118

Roca-Ayats N, Ng PY, Garcia-Giralt N et al (2018b) Functional Characterization of a GGPPS Variant Identified in Atypical Femoral Fracture Patients and Delineation of the Role of GGPPS in Bone-Relevant Cell Types. J Bone Miner Res 33(12):2091-2098

Sarrión P, Sangorrin A, Urreizti R et al (2013) Mutations in the EXT1 and EXT2 genes in Spanish patients with multiple osteochondromas. Sci Rep 3:1346

Shane E, Burr D, Abrahamsen B et al (2014) Atypical subtrochanteric and diaphyseal femoral fractures: second report of a task force of the American Society for Bone and Mineral Research. J Bone Miner Res $29(1): 1-23$

Sidransky E (2004) Gaucher Disease: Complexity in A 'simple' disorder. Mol Genet Metabol 83(1-2): 6-15

Stickens D, Evans GA (1998) A sugar fix for bone tumours? Nat Genet $19(2): 110-111$ 
Sum M, Huskey M, Diemer K, et al. (2013) TNSALP mutation analysis in women with atypical femoral fracture and bisphosphonate therapy for osteoporosis. J Bone Miner Res 28(S1):S295

Tiscornia G, Vivas EL, Izpisúa Belmonte JC (2011) Diseases in a Dish: Modeling Human Genetic Disorders Using Induced Pluripotent Cells. Nat Med 17(12):1570-1576

van Dussen L, Lips P, Everts VE et al (2011) Markers of bone turnover in Gaucher disease: modeling the evolution of bone disease. J Clin Endocrinol Metab 96(7):2194-2205

von Zglinicki T, Petrie J, Kirkwood TB (2003) Telomere-driven replicative senescence is a stress response. Nat Biotechnol 21(3):229-230

Wuyts W, van Hul W (2000) Molecular Basis of Multiple Exostoses: Mutations in the EXT1 and EXT2 Genes. Hum Mutat 15(3):220-227

Zhang P, Lu H, Peixoto RT et al (2018) Heparan Sulfate Organizes Neuronal Synapses through Neurexin Partnerships. Cell 174(6):1450-1464

Zheng HF, Forgetta V, Hsu YH et al (2015) Whole-genome sequencing identifies EN1 as a determinant of bone density and fracture. Nature 526(7571):112-117

Zhou S-F, Liu J-P, Chowbay B (2009) Polymorphism of human cytochrome P450 enzymes and its clinical impact. Drug Metab Rev 41:89-295 


\section{Figure Legends}

Fig. 1. Analysis of a pedigree with a mutation in the EXT2 gene. a) segregation analysis of EXT2 polymorphic markers. A-F refer to positions in the map shown in $b$. b) position of the polymorphic markers (A-F) genotyped in $a$, in relation to EXT2 exons. c) MLPA analysis of the DNA of the proband, her parents and her affected aunt, showing the heterozygous deletion of EXT2 exons 10-16 in the affected individuals.

Fig. 2. a) Schematic diagram of the induction of Mesenchymal Stromal Cells (MSC) including 4 main steps: Embryonic Body (EB) formation, cell outgrowth from EBs on Matrigel-coated dishes, cell dissociation (3X) and monolayer culture b) Percentage of MSC in each phase of the cell cycle for the indicated genotypes. c) GBA1 activity in cells of both N370S/N370S and WT genotypes, at the indicated time points of the osteoblastic differentiation process. Results are shown as mean of GBA1 activity \pm Standard Deviation (SD). GBA1 activity is expressed in nanomoles 4-Methylumbelliferone (MU) $\mathrm{x} \mathrm{mg}^{-1}$ protein $\mathrm{x}$ hour ${ }^{-1}$. Statistical differences $\left({ }^{* * *}\right)$ p-value $<0.001$, using Student's t-test. See Supplementary Material and Methods for further details. 
Fig. 3. Relative mRNA expression of a) $A L P L$ and b) RUNX2 genes during the osteoblastic induction (time points: 0 (MSC), 7, 14 and 21 days) in WT and GD (N370S/N370S) cells. Hypoxanthine phosphoribosyltransferase (HPRT) was used as a housekeeping gene. Data represent the mean values (9 replicates) normalized by WT day 0 (=WT MSC) \pm SD. Statistical differences $\left({ }^{* *}\right)$ p-value $<$ $\left.0.01,{ }^{* * *}\right)$ p-value $<0.001$ and not significant (n.s) using Student's t-test. See Supplementary Material and Methods for further details.

Fig 4. Histochemical assays at $0,7,14$ and 21 days of the osteoblastic differentiation process in both, WT and GD cells. a) alizarin red staining for calcium deposits, b) von Kossa staining for phosphate deposits. See Supplementary Material and Methods for further details.

Fig. 5. a) Schematic representation of the co-culture experiment where the osteoclastogenesis of healthy monocytes induced by WT or GD iPSC-derived MSC/osteoblasts can be evaluated. A membrane separates the well in two compartments. The upper one contains the WT or GD iPSC-derived Mesenchymal Stromal Cells (MSC) or Osteoblasts (OB) and the bottom one contains the peripheral blood $\mathrm{CD} 14^{+}$monocytes from the same healthy female 
donor. Molecules, such as RANKL, but not cells can cross the membrane. b) TRITC-Phalloidin staining (in grey) for the actin cytoskeleton and 4',6-diamidino2-phenylindole (DAPI) staining (in green) for the nuclei. c) Tartrate resistant acid phosphatase (TRAP) staining (in purple/pink) as a specific marker for osteoclasts. d) Size and e) number of nuclei per osteoclast analyzed. The letters in the $x$ axis: $a, b, c, d$, e and $f$ correspond to different healthy female donors, whose monocytes were distributed in two identical parts, one to assess osteoclastogenesis generated by the WT cells and the other for the generated by the GD cells. Results are showed as median \pm confidence interval. Statistical differences $\left({ }^{*}\right)$ p-value $<0.05,\left({ }^{* *}\right) p$-value $<0.01,\left({ }^{* * *}\right) p$-value $<; 0.001$ and n.s, using Mann-Whitney-Wilcoxon test. See Supplementary Material and Methods for further details.

Fig 6. a) Radiograph of an atypical femoral fracture. Note the transverse fracture line that becomes oblique as it progresses (white arrow). LC: focal thickening of the lateral cortex; MS: medial spike. b) Proteins found mutated in AFF patients (in bold) in the context of bone tissue. c) Mevalonate pathway, indicating the steps affected by bisphosphonates (BPs, in red) and the position of GGPPS (circled in green). 


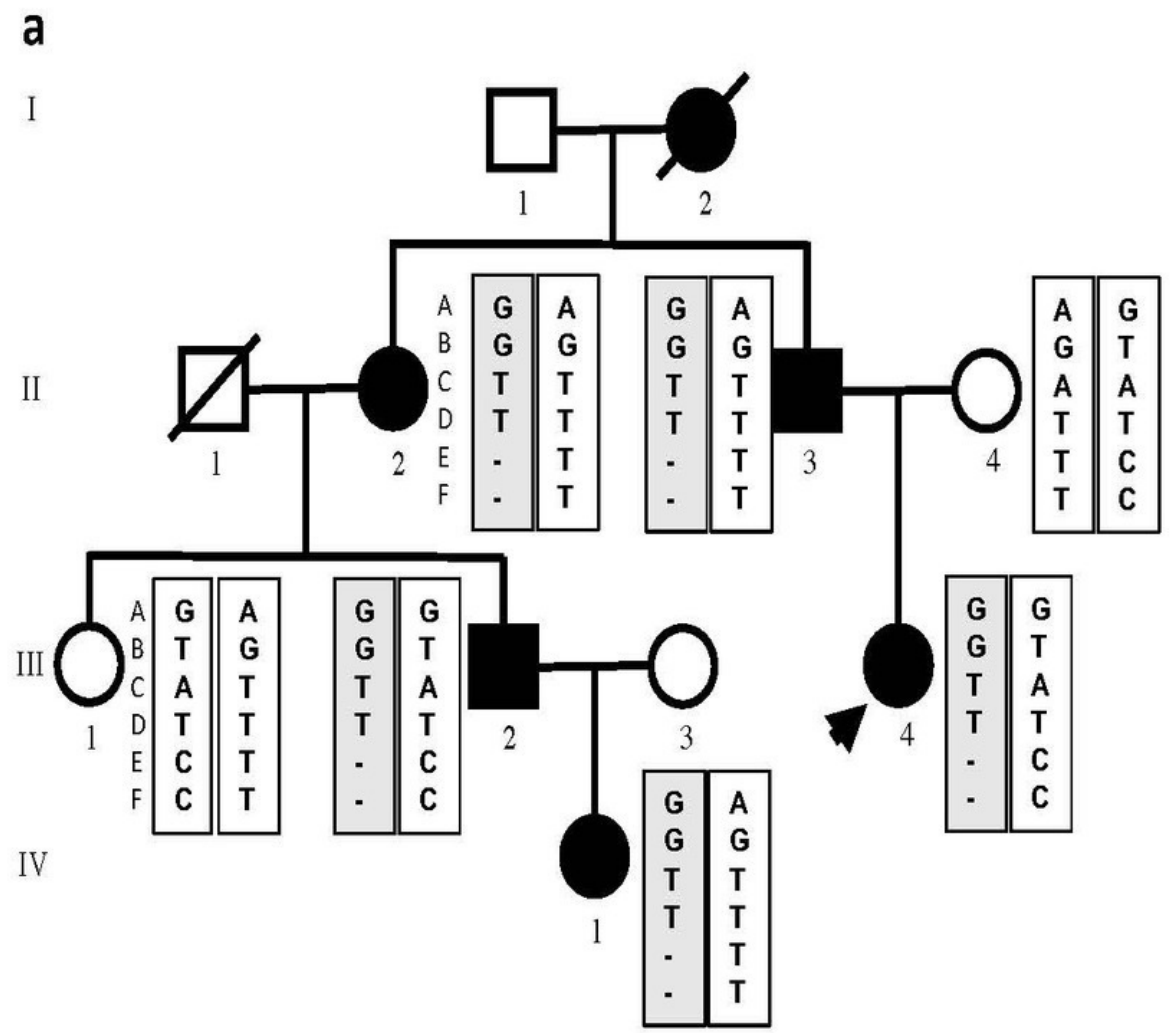

b

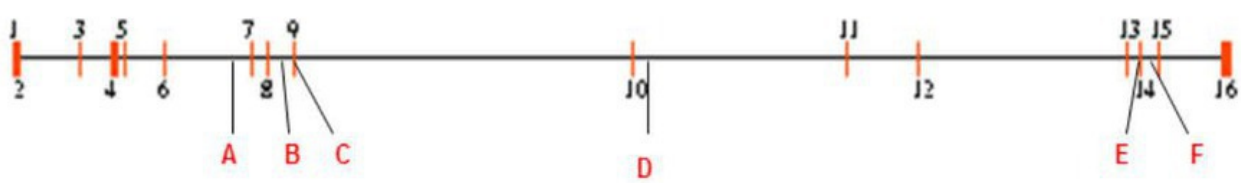

C

Proband (III-4)

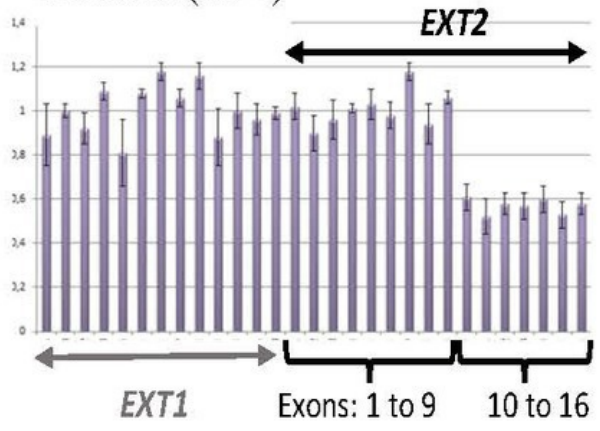

Healthy mother (II-4)

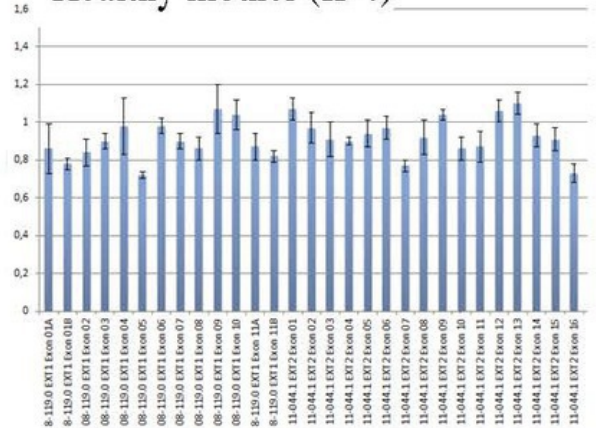

Afected father (II-3)

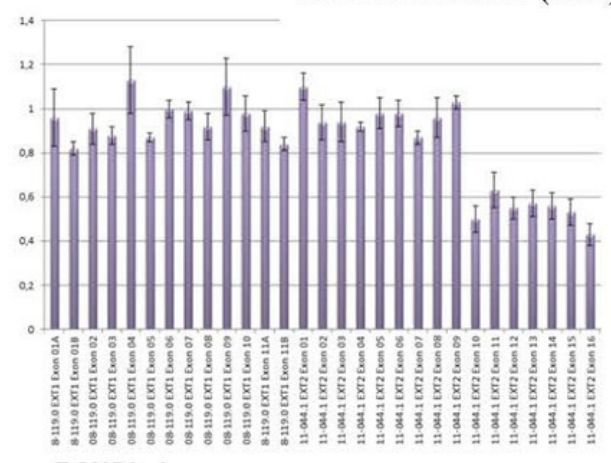

Afected aunt (II-2)

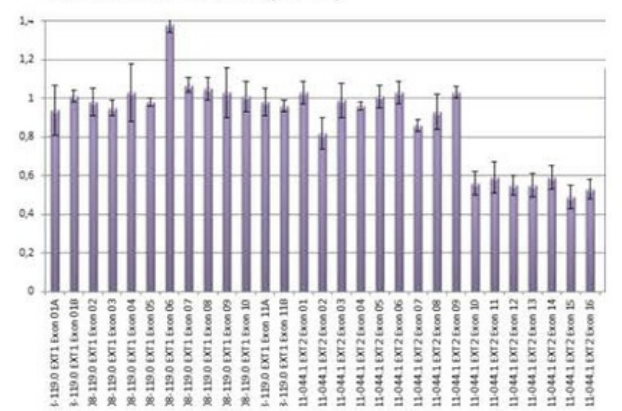


Fig 2

a

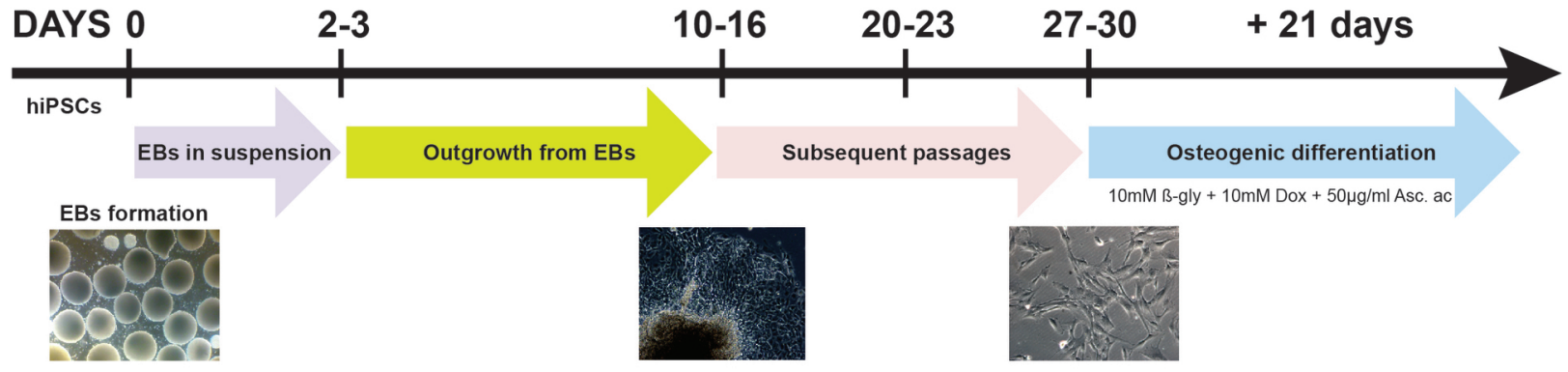

MSC-like cells

(+): CD73, CD90. CD105

$(-):$ D 34, CD45

b

$$
\begin{aligned}
& \text { G2/M phase } \\
& \text { S phase } \\
& \text { G0/G1 phase }
\end{aligned}
$$

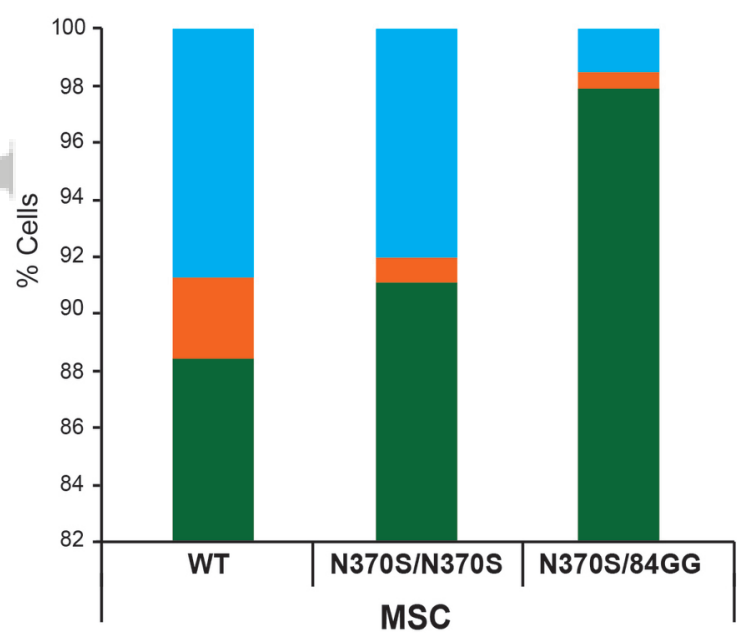

C

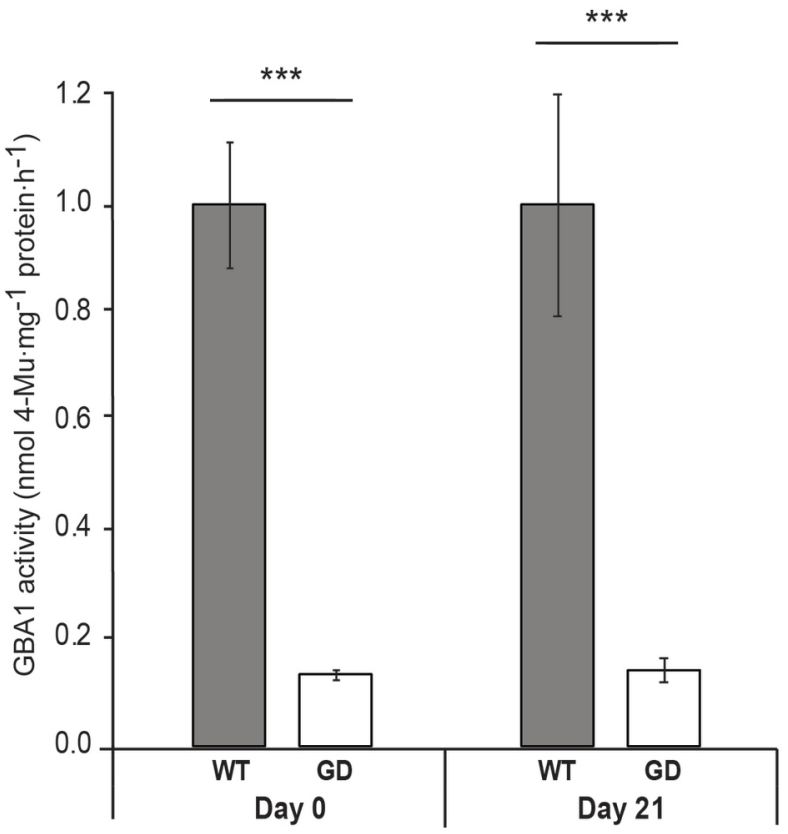

JIMD_12097_Fig.2.jpg 
Fig 3

a

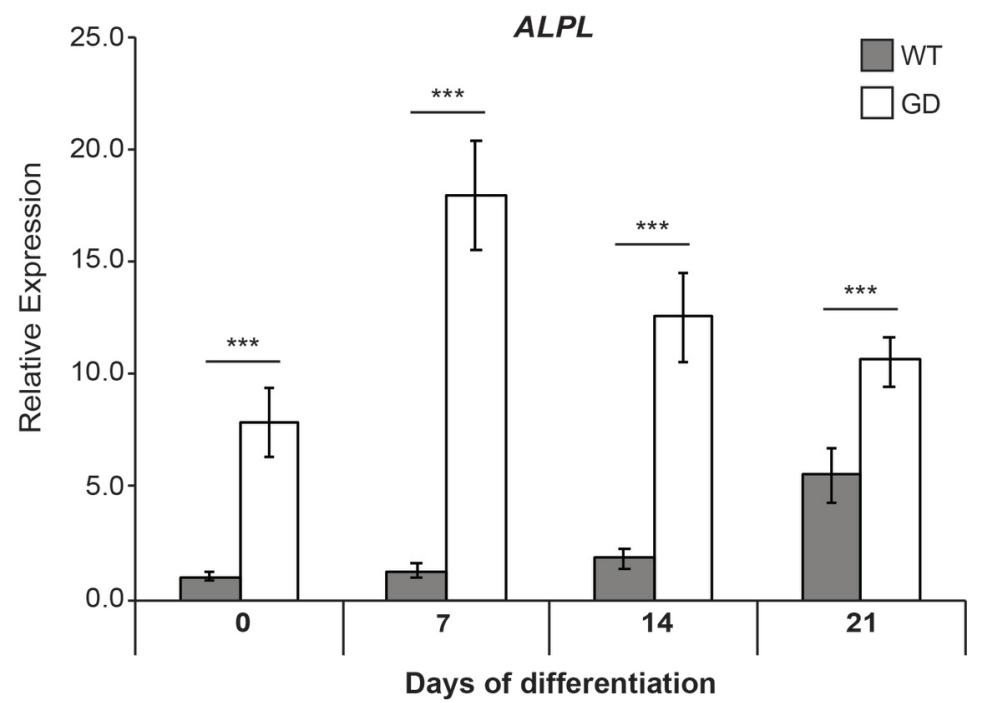

b

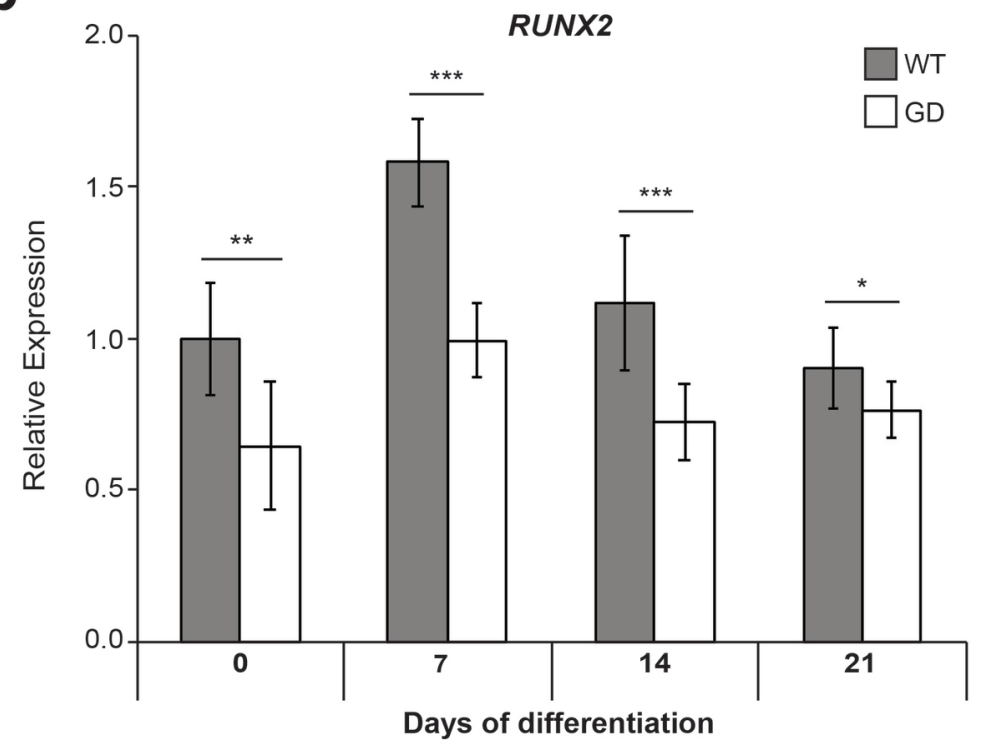

JIMD_12097_Fig.3.jpg 
Fig 4

a
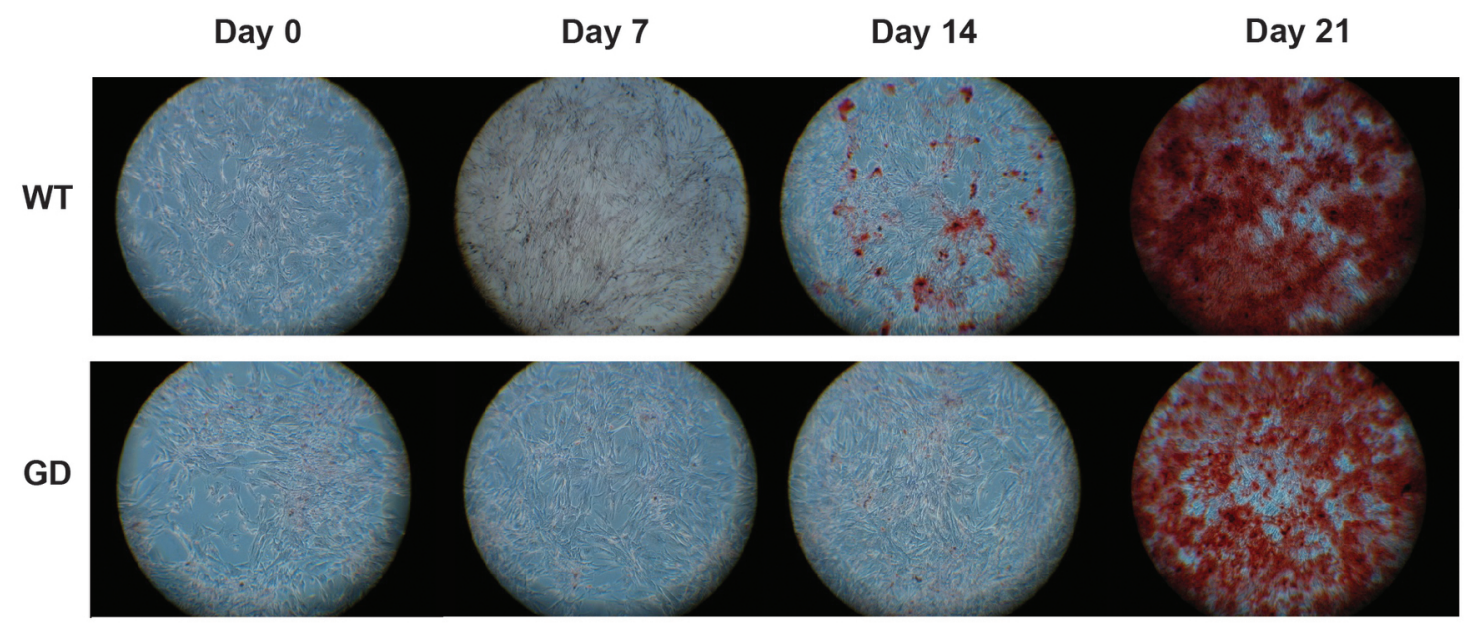

b

Day 0

Day 7

Day 14

Day 21
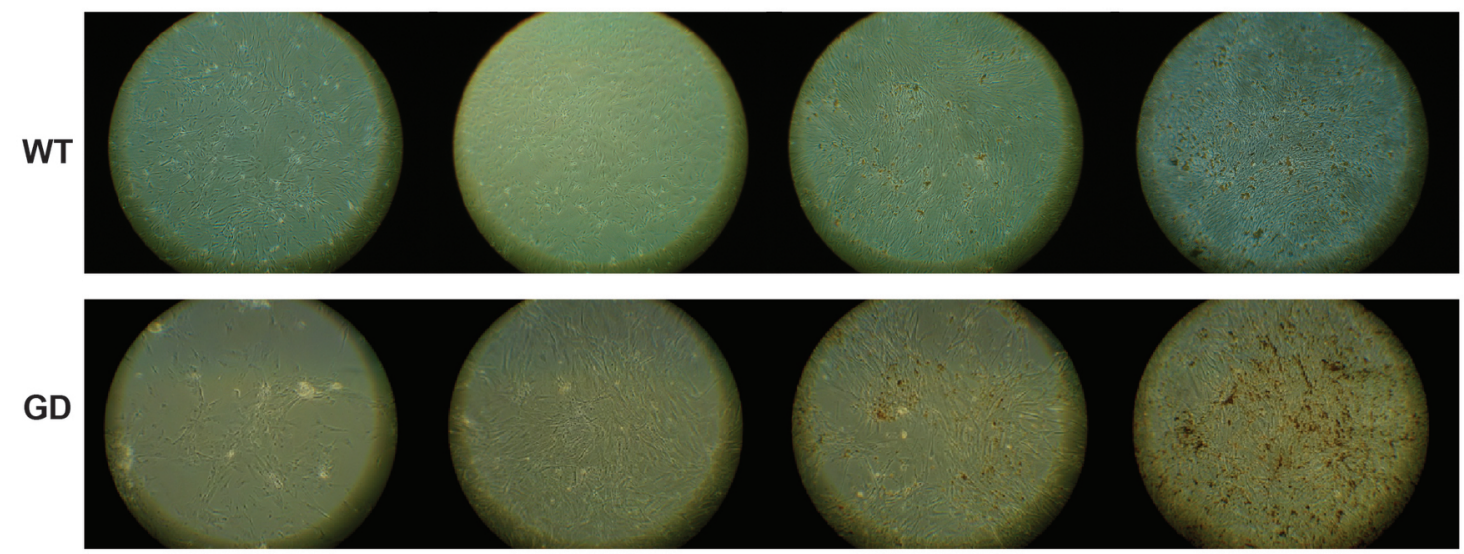

JIMD_12097_Fig.4.jpg 
Fig 5

a
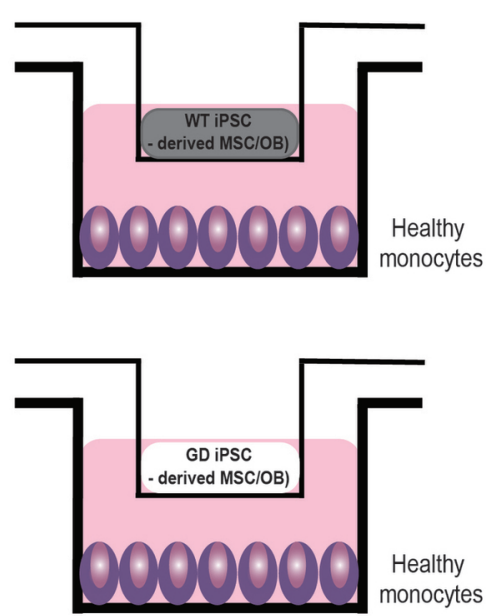

b

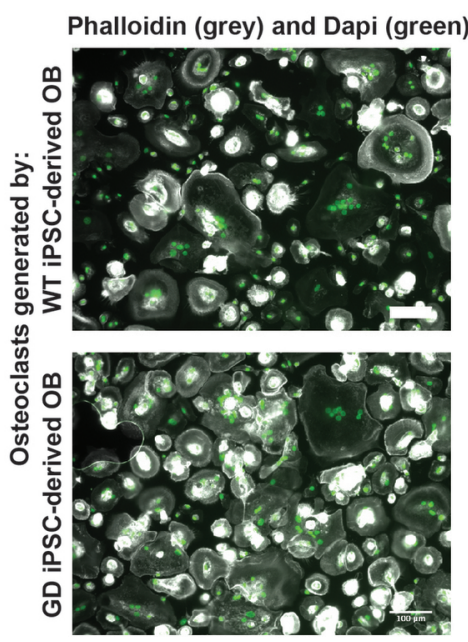

C

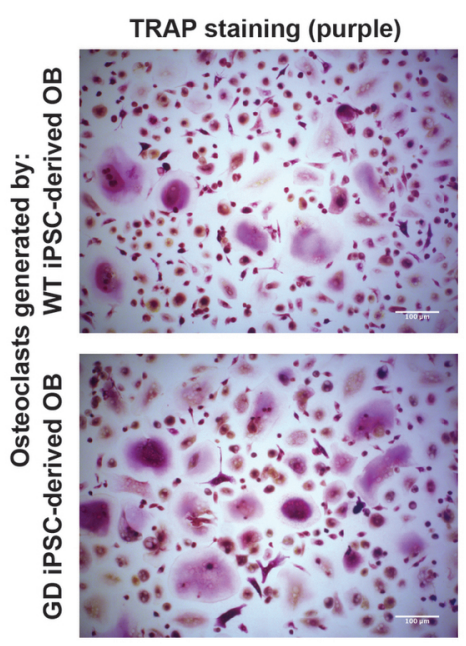

d

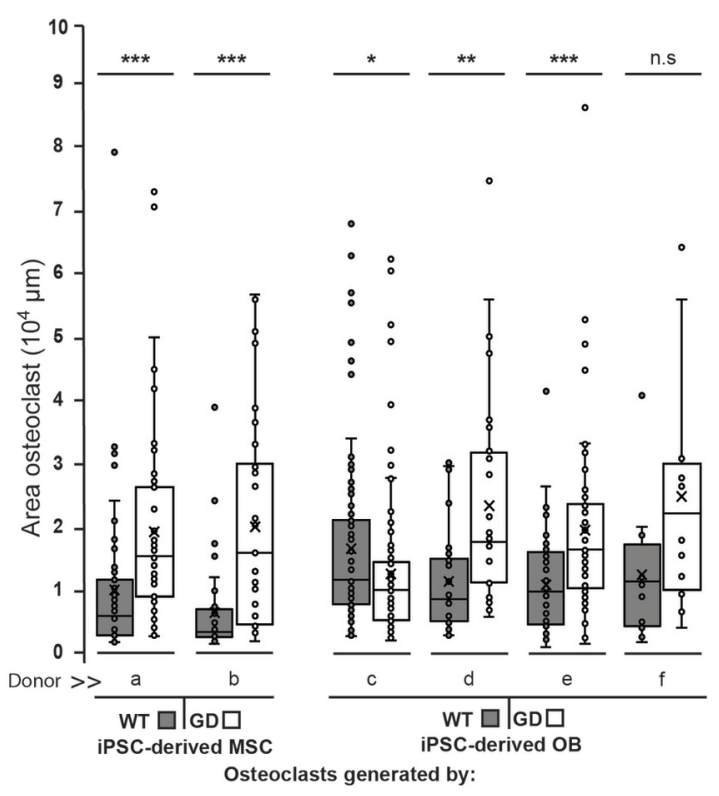

e

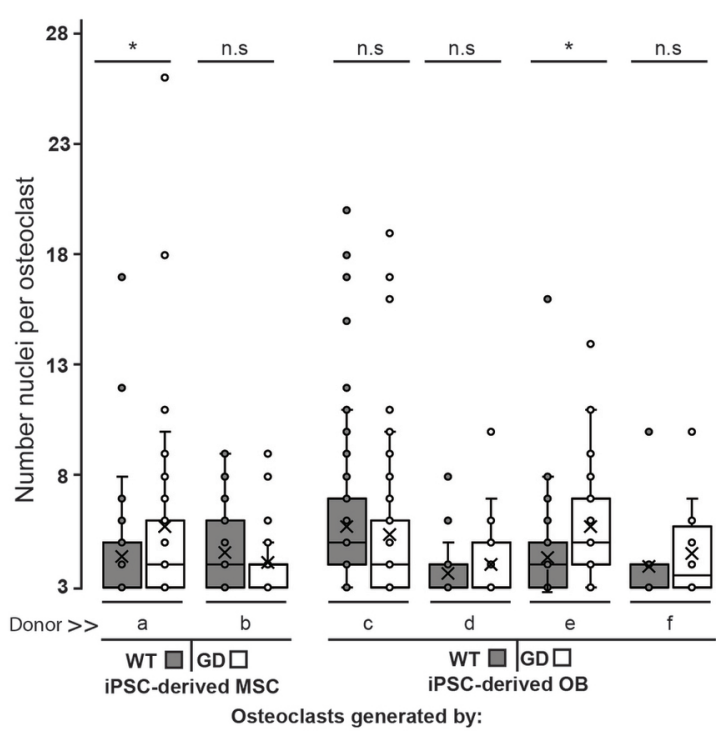

JIMD_12097_Fig.5.jpg 

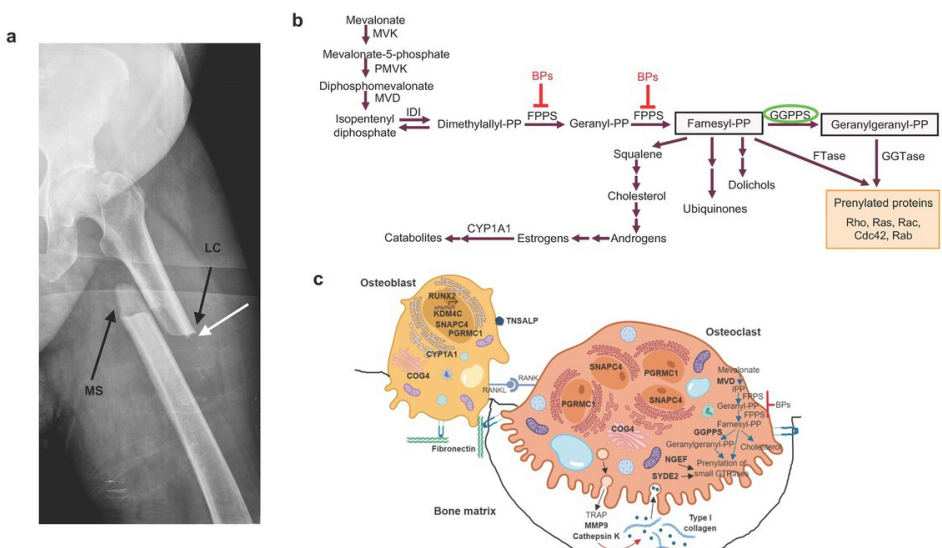

c

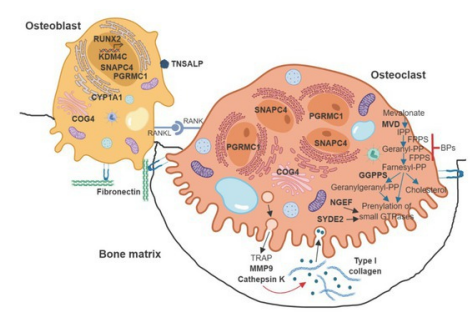

JIMD_12097_Fig.6.jpg

This article is protected by copyright. All rights reserved. 


\section{MATERIALS AND METHODS}

\section{Culture of the induced Pluripotent Stem Cells (iPSC)}

The generation of the human Gaucher disease (GD) induced Pluripotent Stem Cells (iPSC) lines with the N370S/N370S (Panicker et al. 2012), G202R/L444P (Tiscornia et al. 2011) and N370S/84GG (Park et al. 2008) genotypes were previously described. Wild-type (WT) iPSC cells were generated by our group (Canals et al. 2015). They were maintained in human embryonic stem cells (HES) medium, consisting of knockout Dulbecco's modified Eagle medium (KODMEM; Gibco, Cat. No. 10829018) supplemented with $20 \%$ KO-serum replacement (Gibco, \#10828010), 2mM Glutamax (Gibco, \#35050061), 50mM 2-mercaptoethanol (Gibco, Cat. No. 21985023), 1\% Minimum Essential Media (MEM) non-essential amino acids (Gibco, Cat. No. 11140035), 1\% penicillin/streptomycin (Gibco, Cat. No. 15140122) and 10ng/ml basic fibroblast growth factor (bFGF, Peprotech, Cat. No. 100-18B). Cultures were maintained on top of a human foreskin fibroblast feeder layer at $37^{\circ} \mathrm{C}$ and $5 \% \mathrm{CO}_{2}$. The culture medium was changed every day.

\section{Induction of mesenchymal stem cell (MSC)-like cells}

Once feeder-iPSC colonies, maintained on Matrigel ${ }^{\mathrm{TM}}$ (BD Biosciences, Cat. No. 354277) and fed with HESc (HES conditioned with mouse embryonic fibroblast and supplemented with $4 \mathrm{ng} / \mathrm{ml}$ bFGF) medium, were at approximately $80 \%$ confluence in $100 \mathrm{~mm}$ dishes, the protocol of embryonic body (EB) formation was started. Culture medium was aspirated and colonies were washed twice with phosphate buffered saline (PBS). Then, 0.05\% TrypsinEDTA (Gibco, Cat. No. 25300104) was added to dissociate the colonies in small clusters. The resulting suspension was transferred to $50 \mathrm{ml}$ falcon tubes and the desired amount of HESc medium was added. A volume of $100-120 \mu l$ of resuspended cells was loaded into each well of $96-\mathrm{V}$ bottom-well microtiter plates and centrifuged at $800 \mathrm{~g}$ for $10 \mathrm{~min}$ to form iPSC aggregates in HESc, that were placed at $37^{\circ} \mathrm{C}, 5 \% \mathrm{CO}_{2}$ during 2-3 days. After this growing period, the EBs were obtained and they were kept in suspension for 2-3 more days. The formed EBs were transferred into 6-well plates coated with Matrigel and

This article has been accepted for publication and undergone full peer review but has not been through the copyediting, typesetting, pagination and proofreading process which may lead to differences between this version and the Version of Record. Please cite this article as doi: 10.1002/jimd.12097 
cultured in EB medium (consisting of KO-DMEM supplemented with $10 \% \mathrm{KO}$ serum Replacement, 2mM Glutamax, 50mM 2-beta-marcaptoethanol, 1\% MEM non-essential aminoacids and $1 \%$ penicillin/streptomycin) to let their outgrowth reach the confluence (it takes around 10-16 days). Then, the EB outgrowths were dissociated using 0.25\% Trypsin-EDTA (Gibco, Cat. No. 25200056). The cells were subsequently resuspended in EB medium and seeded at a ratio of 1:2. Subsequently, confluent cells were dissociated two more times with $0.25 \%$ Trypsin-EDTA to completely remove the pluripotent cells in our culture and to obtain homogenous monolayer of mesenchymal-like cells (this passage was considered as passage 0 of MSC-like cells and the cells were ready to their subsequent validation by flow cytometry). In this moment, most of the cells in culture had a fibroblast-like appearance.

\section{Flow cytometric analysis of cell surface antigens}

Expression of the cell surface antigen profile of MSCs was characterized using flow cytometry. MSC-like cells were harvested using $0.05 \%$ Trypsin/EDTA. After neutralization, single-cell suspension was washed in cold PBS. Then, around $1 \times 10^{6}$ cells in $100 \mu$ l PBS were incubated with each monoclonal antibody following the manufacturer's instructions. The following conjugated mouse antihuman antibodies were used in the analyses: brilliant violet ${ }^{\mathrm{TM}} 421$-conjugated against CD34, allophycocyanin (APC)-cyanine ${ }^{\mathrm{TM}} 7$-conjugated against CD45 as a non-specific MSC markers and APC-conjugated against CD73, fluorescein isothiocyanate-conjugated against CD90, phycoerythrin-conjugated against CD105 as specific MSC markers (all from BD Pharmingen, Cat. No. 562577, $557833,560847,555595,560839$, respectively). After 30 min incubation on ice, cells were washed twice with cold PBS, followed by centrifugation at $400 \mathrm{~g}$ for 5 min. The pellet was resuspended with $300-500 \mu$ l PBS. Unstained cells were used as a control. Samples were run on a Becton Dickinson Facscanflow cytometric system (BD Biosciences), and analysis was completed using a FlowJo Software (Tree Star Inc.).

\section{Culture of MSC-like cells}

MSC-like cells were maintained in $0.1 \%$ (W/V) gelatin-coated T75 flask with MSC medium, consisting of alpha-MEM (Gibco, Cat. No. 22561021), 
supplemented with $10 \%$ fetal bovine serum (FBS; Hyclone Laboratories Inc., Cat No. SH3007103), 2mM Glutamax and 1\% penicillin/streptomycin. Culture medium was changed every 3 days, and when the cells reached $80-90 \%$ confluence, they were subcultured using $0.05 \%$ Trypsin-EDTA.

\section{Cell cycle analysis}

Cell cycle analysis was assessed on MSC at passages 3-4. Cells were trypsinized and fixed with $70 \%$ ethanol for 2 hours. After ethanol removal, cells were washed twice with PBS and the pellet was incubated in a PBS solution containing $0.1 \%$ Triton-X-100, $10 \mu \mathrm{g} / \mathrm{ml}$ propidium iodide (Sigma, Cat. No. P4170), and 2mg/ml Ribonuclease A (Sigma, Cat. No. R6513). Cell cycle analysis was performed by flow cytometry, and the proportion of cells in the G0/G1, S, and G2/M phase was calculated using the Cell Quest Pro software (BD Biosciences).

\section{Induction of osteogenic differentiation}

For osteogenic differentiation, cells were seeded in 6-well, 12-well, and 24-well culture dishes or $100 \mathrm{~mm}$ dishes depending on the specific experiment. When cells achieved $80 \%$ confluence, osteogenic differentiation was induced with alpha-MEM (Gibco) supplemented with $10 \%$ FBS, $50 \mu \mathrm{g} / \mathrm{ml}$ ascorbic acid (Sigma, Cat. No. BP461), 10mM $\beta$-glycerophosphate (Sigma, Cat. No. G9422), 100nM dexamethasone (Sigma, Cat. No. D4902), and 1\% penicillin/streptomycin. Medium was changed every 3 days during 21 days.

\section{Histochemical assays}

For histochemical staining, cultures were washed twice with PBS and fixed with $4 \%$ (W/V) paraformaldehyde (PFA) for $10 \mathrm{~min}$ at room temperature and rinsed with double-distillated water.

For calcium deposits, the fixed cultures were covered with a 40mM Alizarin Red solution, $\mathrm{pH}=4.1$ (adjusted with $10 \% \mathrm{NH}_{4} \mathrm{OH}$ ) for 20 min and then rinsed with double-distilled water. Calcium deposits stained red.

Phosphate deposits were assessed by the von Kossa technique. The fixed cultures were covered with a $1.0 \%$ (W/V) silver nitrate solution and kept for 1 
hour under ultraviolet light. After rinsing twice with double-distilled water, a 5.0\% (W/V) sodium thiosulphate was added for $2 \mathrm{~min}$ and then cultures were washed again. Phosphate deposits stained black. The cells were stored in Tris-buffered at light protected until photos were taken.

\section{Glucocerebrosidase (GBA1) activity measurement}

GBA1 enzyme activity measurement was performed at the MSC stage (day 0) and at 21st day of differentiation, using 4-methylumbelliferyl (4-MU) glucopyranoside as artificial substrate. Cells (seeded in 24-well culture dishes) were washed twice with PBS and $100 \mu \mathrm{l}$ of $5 \mathrm{mM} 4-\mathrm{MU}$ in $0.1 \mathrm{M}$ acetate buffer (pH 5.2) was added to each well, up to a total volume of $160 \mu l$. Then the plates were incubated for 1 hour at $37^{\circ} \mathrm{C}$ and enzyme reaction was stopped with $2 \mathrm{ml}$ of $100 \mathrm{mM}$ glycine $/ \mathrm{NaOH}, \mathrm{pH} 10.7$. The released fluorescence was measured at $\lambda_{\mathrm{ex}}: 355 \mathrm{~nm} ; \lambda_{\mathrm{em}}: 460 \mathrm{~nm}$. The experiment was performed 3 times with at least 6 replicates per each point (stages 0 and 21 days).

\section{Real-time reverse transcription-polymerase chain reaction}

Total RNA was extracted from cells plated in $100 \mathrm{~mm}$ dishes using the High Pure RNA Isolation Kit (Roche Applied Science, Cat. No. 11828665001). cDNA was prepared using the High Capacity cDNA Reverse Transcription kit (Applied Biosystems, Cat. No. 4368814) following the manufacturer's instructions. Realtime PCR was performed on a LightCycler 480 II system using the Universal Probe Library (Roche Applied Science, Cat. No. 05015243001) according to the manufacturer's instructions. The specific primers, listed in table S1, for hypoxanthine phosphoribosyltransferase (HPRT), tissue-nonspecific alkaline phosphatase $(A L P L)$ and runt-related transcription factor 2 (RUNX2) were designed using the Universal ProbeLibrary Assay Design Center software (Roche Applied Science, www.roche-applied-science.com). All the reactions were run in triplicate and 3 independent experiments with 3 replicates each were performed. The expression of HPRT was used as housekeeping gene to normalize the expression of $A L P L$ and $R U N X 2$. 
Table S1. List of primer sequences and Universal Probe number (Roche Applied Science) used for RT-PCR analysis

\begin{tabular}{cccc}
\hline Gene & $\begin{array}{c}\text { Forward (Fw) and Reverse (Rv) primer } \\
\left(\mathbf{5}^{\prime} \text { to } \mathbf{3}^{\prime}\right)\end{array}$ & $\begin{array}{c}\text { Amplicon } \\
\text { size }\end{array}$ & $\begin{array}{c}\text { Universal probe } \\
\text { number }\end{array}$ \\
\hline ALPL & Fw - GACGGACCCGTCACTCTC & $109 \mathrm{bp}$ & \#77 (Cat. No. \\
& Rv- GTGCCCGTGGTCAATTCT & & $04689003001)$ \\
RUNX2 & Fw- GGCGCATTTCAGATGATGA & $95 \mathrm{bp}$ & \#87 (Cat. No. \\
& Rv- GCCCAGTTCTGAAGCACCT & & $04689127001)$ \\
HPRT & Fw- GACCAGTCAACAGGGGACAT & $95 \mathrm{bp}$ & $\# 22$ (Cat. No. \\
& Rv- GTGTCAATTATATCTTCCACAATCAAG & & $04686969001)$
\end{tabular}

\section{Osteoclasts formation assay}

Human peripheral blood mononuclear cells (PBMC) were isolated from healthy female donors in accordance with the approved ethical procedures of Clinical Research Ethics Committee of Parc de Salut MAR. Monocytes, the osteoclast precursors, were isolated from peripheral blood mononuclear cells (PBMC) using Human Monocyte Enrichment Cocktail RosetteSepTM (Stemcell Technologies, Cat. No. 15028) following the manufacturer's instructions. After isolating the monocyte fraction, the CD14 marker, strongly expressed on monocytes, was determined by flow cytometry using a fluorochrome conjugated anti-CD14 antibody, usually obtaining a culture purity of $90 \%$ CD14+ cells. Peripheral blood $\mathrm{CD} 14^{+}$monocytes were seeded at $5 \times 10^{5}$ cells on $10 \mathrm{~mm}$ glass cover slips (Marienfeld, Cat. No. 0111500) in 24-well plates. These cells were incubated for 2 hours at $37^{\circ} \mathrm{C}$ in $5 \% \mathrm{CO}_{2}$ in complete medium, consisting in the following: alpha-MEM, supplemented with 10\% FBS, 2mM Glutamax, 1\% penicillin/streptomycin and $25 \mathrm{ng} / \mathrm{ml}$ of recombinant human macrophage colony stimulating factor M-CSF(R\&D systems, Cat. No. 216-MC). Non-adherent cells were washed out and adherent cells were used for the osteoclast formation assays. The M-CSF in the media was kept during 3 days to induce monocytes to differentiate into preosteoclasts. On the third day, WT or GD iPSC-derived Mesenchymal Stromal Cells (MSC) or Osteoblasts (OB) were seeded at $6.5 \mathrm{~mm}$ Transwell with $0.4 \mu \mathrm{m}$ pore polycarbonate membranes (Corning, Cat. No. 3413) that were inserted in the 24-well plate containing the preosteoclasts. All the cultures were maintained until the generation of multinucleated giant cells, 
which occurred at around 24-28 days of culture. Culture medium was changed every 3 days.

\section{Phalloidin and DAPI staining}

Osteoclasts have a ring structure composed of actin. This actin ring is a hallmark of the degradative capacity of functional osteoclasts, without this actin ring, osteoclasts cannot resorb bone efficiently. The obtained osteoclasts were washed with PBS and fixed with 3.7\% (W/V) PFA for $10 \mathrm{~min}$. Cells were permeabilized with $0.1 \%$ Triton-X-100 in PBS for 3-5 min. The cytoskeletal actin was stained with TRITC-Phalloidin (Sigma, Cat. No. P1951) and cell nuclei with 4',6-Diamidino-2-phenylindole dihydrochloride (DAPI; Sigma, D9542). Images were acquired with an SP2 confocal system (Leica). In each multinucleated cell (more than 3 nuclei) the number of nuclei was counted and the area was measured using the using Fiji/lmageJ sofware.

\section{Tartrate-resistant acid phosphatase (TRAP) histochemical staining}

The obtained osteoclasts were washed with PBS and the TRAP staining was performed using a leukocyte acid phosphatase kit (Sigma, Cat. No. 387) following the manufacture's recommended protocol. TRAP-positive osteoclasts were visualized under a phase-contrast microscope.

\section{Statistical analysis}

Statistical differences $\left(^{*}\right)$ p-value $<0.05$, $\left({ }^{* *}\right)$ p-value $<0.01$, $\left({ }^{* * *}\right)$ p-value $<; 0.001$ and not significant (n.s), were assessed using two-tailed unpaired Student's t-test or Mann-Whitney-Wilcoxon test, as appropriate. 